\title{
Brain Derived Neurotrophic Factor and Exercise
}

\author{
Daniel Jaffe*, Jennifer Hewit, Kelly Hamilton, Jessica Burkett, Josiah Luce and Alexander Bedard \\ Department of Physical Education, United States Military Academy, USA
}

*Corresponding author: Daniel Jaffe, Department of Physical Education, United States Military Academy, West Point, 10996, NY, USA

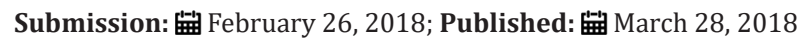

\section{Editorial}

Brain-derived neurotrophic factor (BDNF), a dimeric protein found throughout the brain, promotes the survival of nerve cells by playing a role in the growth, maturation, and maintenance of cells [1]. Along with supporting the survival of existing neurons, BDNF encourages differentiation and growth of new neurons and synapses [2,3]. In the brain the hippocampus, cortex, and basal forebrain, which are vital to learning, memory, and higher thinking, are all locations where BDNF is active [4]. A number of stimuli have been found to increase BDNF gene regulation including light in the visual cortex, osmotic in the hypothalamus, electrical in the hippocampus, and exercise in the hippocampus [5].

Changes in the levels and activities of BDNF have also been described in a number of neurodegenerative disorders including Huntington's disease, Alzheimer's disease, and Parkinson's disease [1]. Additionally, as it plays a role in brain development and plasticity, BDNF is also implicated in psychiatric diseases such as depressive disorder, schizophrenia, addiction, and Rett syndrome [6]. Treatments, such as antidepressants, increase the expression of BDNF influencing behavior and feelings of helplessness [6]. BDNF is also identified as a key component of the hypothalamic pathway that controls energy homeostasis and body weight. Low levels of BDNF accompany impaired glucose metabolism [7]. This means that decreased BDNF levels may be a pathogenic factor involved not only in dementia and depression, but also in type 2 diabetes, potentially explaining the clustering of these conditions [7].

Management of Alzheimer's disease (AD), particularly the behavioral problems associated with the dementing disorder, can be significantly affected through the employment of physical activity and antidepressant treatment [1]. A study conducted on the hippocampus of rats found that antidepressant treatment and physical activity have an additive effect on BDNF mRNA expression within the brain. Throughout the 20-day experiment, the rats were injected daily with $15 \mathrm{mg} / \mathrm{kg}$ of imipramine, a nerve pain medication and antidepressant, or $7.5 \mathrm{mg} / \mathrm{kg}$ of tranylcypromine, an antidepressant. Rats assigned to the exercise group were given access to running wheels for the length of the experiment [1].
Following the 20-day experiment, the BDNF mRNA levels were evaluated in various cell areas of the hippocampus. The study found that the combination of the antidepressant treatment and physical activity led to a substantial potentiation of BDNF mRNA levels within the dentate gyrus, which were well-above the levels found with each intervention alone. The results of the study provide evidence to bolster the argument that physical exercise can serve as an enhancement for treatment response to antidepressants [1].

A second study, also conducted with rats, tested the hypothesis that BDNF in the hippocampus would create an antidepressant effect in the learned helplessness (LH) and forced swim test (FST), which are both behavioral models of depression [2]. An injection of BDNF into the dentate gyrus of hippocampus yielded an antidepressant effect in both the LH and FST. This BDNF infusion was considered to be similar in magnitude with repeated administration of a chemical antidepressant. The effects were examined 3-days after a single infusion of BDNF and continued for up to 10-days. The results of this study also confirm the hypothesis that BDNF promotes the therapeutic effects of antidepressant treatment [2].

With this up-regulation of BDNF levels through exercise, several positive health and neural benefits have been revealed. Increased BDNF in rats has been shown to help increase the brain's resistance to damage and neural degeneration that occurs with aging. Being that BDNF helps to increase neural plasticity, increased levels of it would help to keep neurons from dying [8]. Aerobic exercise, such as running has been shown to produce the most robust BDNF response, and varying the intensity and type of exercise has been shown to have an effect on up-regulation of BDNF as well [9]. Moreover, while BDNF levels and its connection to cognitive function have not been clearly mapped, researchers have noted that decreased levels of BDNF are associated with depression. As exercise causes increases in circulating BDNF levels, researchers hypothesize that physical activity could also help to eradicate depression, corroborating research within the realm of psychology that suggests exercise positively affects mood and can decrease depression [8]. 


\section{References}

1. Russo-Neustadt A, Beard RC, Cotman CW (1991) Exercise, antidepressant medications, and enhanced brain derived neurotrophic factor expression. Neuropsychopharmacology 21(5): 679-682.

2. Shirayama Y, Chen ACH, Nakagawa S, Russell DS, Duman RS (2002) Brain-derived neutrophic factor produces antidepressant effects in behavioral models of depression. J Neurosci 22(8): 3251-3261.

3. Huang EJ, Reichardt LF (2009) Neurotrophins: roles in neuronal development and function. Annu Rev Neurosci 24: 677-736.

4. Yamada K, Nabeshima T (2003) Brain-derived neurotrophic factor/TrkB signaling in memory processes. J of Pharm Sci 91(4): 267-270.

5. Binder DK, Scharfman HE (2004) Brain-derived neurotrophic factor Growth Factors 22(3): 123-131.
6. Autry AE, Monteggia LM (2012) Brain-derived neurotrophic factor and neuropsychiatric disorders. Pharm Rev 64(2): 238-258.

7. Krabbe KS, Nielsen AR, Krogh-Madsen R, Plomgaard P, Rasmussen P, et al. (2007) Brain-derived neurotrophic factor (BDNF) and type 2 diabetes. Diabetologia 50(2): 431-438.

8. Oliff HS, Berchtold NC, Isackson P, Cotman CW (1998) Exercise-induced regulation of brain-derived neurotrophic factor (BDNF) transcripts in the rat hippocampus. Brain Res Mol Brain Res 61(1-2): 147-153.

9. Ferris LT, Williams JS, Shen C (2007) The effect of acute exercise on serum brain-derived neurotrophic factor levels and cognitive function. Med Sci Sports Exerc 39(4): 728-734. (c) 1 Creative Commons Attribution 4.0

For possible submissions Click Here

\section{Submit Article}

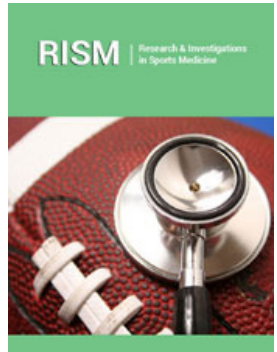

Research \& Investigations in Sports Medicine

Benefits of Publishing with us

- High-level peer review and editorial services

- Freely accessible online immediately upon publication

- Authors retain the copyright to their work

- Licensing it under a Creative Commons license

- Visibility through different online platforms 